\title{
ON SCHREIER SYSTEMS IN FREE GROUPS
}

\author{
BY \\ MARSHALL HALL, JR. AND TIBOR RADO
}

\section{Introduction.}

1.1. Let $\mathfrak{F}$ be the free group over the (free) generators $S_{1}, \cdots, S_{j}, \cdots$, where the number of these generators is either finite or countably infinite. A (finite or countably infinite) sequence of elements $g_{1}, \cdots, g_{i}, \cdots$ of $\mathfrak{F}$, each $g_{i}$ being assumed to be written in reduced form, will be termed a Schreier system provided that the following conditions are satisfied (in the following statements and throughout the paper, $G$ will be used as a generic notation for an element of the sequence $g_{i}$ ).

(a) $g_{1}=1$.

(b) If $G \neq 1$ and $G=S_{f_{1}}^{\epsilon_{1}} \cdots S_{f_{r}}^{\epsilon_{r}}$, where each $\epsilon= \pm 1$ and $G$ is written in reduced form as agreed above, then each of the "beginning sections"

$$
S_{j_{1}}^{\epsilon_{1}}, S_{j_{1}}^{\epsilon_{1}} S_{j_{2}}^{\epsilon_{2}}, \cdots, S_{j_{1}}^{\epsilon_{1}} S_{j_{2}}^{\epsilon_{2}} \cdots S_{j_{r-1}}^{\epsilon_{r-1}}
$$

is also an element of the sequence $g_{i}$.

For example, if $\mathfrak{F}$ is the free group over just two (free) generators which we then denote by $a, b$ instead of $S_{1}, S_{2}$, we have the following instances of Schreier systems:

$$
1, a, a^{2} ; 1, b, b^{2} ; 1, a, b ; 1, a, b, a b, b a ; 1, a, a^{2}, b, b a, b a^{2} .
$$

While these particular Schreier systems are finite, it should be noted that a general Schreier system, as defined above, may be countably infinite.

Let $\mathfrak{U}$ be a subgroup of $\mathfrak{F}$, and let $K_{1}=\mathfrak{U}, K_{2}, \cdots, K_{i}, \cdots$ be the (finite or countably infinite) sequence of left cosets relative to $\mathfrak{U}$. Then Schreier (see [1](1)) proved that it is always possible to select from each $K_{i}$ a "representative" $g_{i}$ such that the representatives $g_{1}, \cdots, g_{i}, \cdots$ constitute what we termed a Schreier system. Assuming that the representatives $g_{i}$ have been so selected, let us denote, for any given element $f \in \mathfrak{F}$, by $\Psi(f)$ the representative $g_{i}$ of the left coset $K_{i}$ that contains $f$. Let us use $G$ as a generic notation for a term of the sequence $g_{i}, S$ as a generic notation for a generator $S_{1}, \cdots$, $S_{j}, \cdots$, and $U$ as a generic notation for an element of $\mathfrak{F}$ that can be written in the form

$$
U=G S[\Psi(G S)]^{-1}
$$

Presented to the Society, September 3, 1947; received by the editors July 2, 1947, and, in revised form, August 13, 1947.

(1) Numbers in brackets refer to the bibliography at the end of the paper. 
For certain choices of $G$ and $S$ we may have $U=1$. Let $U_{1}, U_{2}, \cdots$ be the (finite or countably infinite) sequence of those elements $U$ that are not equal to 1 (each $U \neq 1$ being listed just once). Then Schreier showed that $U_{1}, U_{2}, \cdots$ generate $\mathfrak{U}$ and are in fact free generators of $\mathfrak{U}$. In this manner, Schreier obtained a first general proof of the theorem that every subgroup of a free group is free.

1.2. Let us say that a Schreier system $g_{1}, \cdots, g_{i}, \cdots$ and a subgroup $\mathfrak{U}$ of $\mathfrak{F}$ are associated with each other if each left coset relative to $\mathfrak{U}$ contains exactly one element of the sequence $g_{i}$. According to the results of Schreier outlined above, each subgroup $\mathfrak{U}$ of $\mathfrak{F}$ is associated with at least one Schreier system. In this paper, we shall study the inverse problem: given a Schreier system $g_{1}, \cdots, g_{i}, \cdots$, is there always an associated subgroup $\mathfrak{U}$ of $\mathfrak{F}$ ? We shall find that the answer is in the affirmative if the given Schreier system is finite. For the case of infinite Schreier systems, we are indebted to the referee for comments that led us to recognize the need for some further restriction to insure the existence of an associated subgroup. We shall give a simple condition, to be termed the condition (C) (see 2.7), which is necessary and sufficient for the existence of an associated subgroup. Furthermore, we shall develop a definite process that yields all the subgroups that are associated with a given Schreier system satisfying the condition (C). In the case of finite Schreier systems, condition (C) is readily seen to hold automatically.

1.3. Let $f$ be an element of $\mathfrak{F}$, and let $f=S_{i_{1}}^{\epsilon_{1}} \cdots S_{i_{r}}^{\epsilon_{r}}$ be the reduced expression of $f$ in terms of the generators $S$. Then the number $r$ of factors is termed the length $l(f)$ of $f$, it being understood that for $f=1$ we put $l(f)=0$. Let now $\mathfrak{U}$ be a subgroup of $\mathfrak{F}$ and let $g_{1}, \cdots, g_{i}, \cdots$ be an associated Schreier system (see 1.2). For each $i$, let $K_{i}$ be the left coset (relative to $\mathfrak{u}$ ) that contains $g_{i}$. Then the Schreier system will be termed shortest relative to $\mathfrak{u}$ provided that for every $i$ we have the inequality $l\left(g_{i}\right) \leqq l(f)$ for every element $f \in K_{i}$. Schreier proved that for every subgroup $\mathfrak{U}$ of $\mathfrak{F}$ there exists a shortest associated Schreier system. However, he did not make any further use of this extremal property. We shall discuss in some detail the implications. of the extremal property just mentioned, and we shall obtain certain interesting relationships to the work of Nielsen [2] which preceded the work of Schreier but apparently did not attract the attention it deserves. In particular, we shall obtain a generalization of an important result of Nielsen concerning free generators of minimal length, for subgroups $\mathfrak{U}$ of $\mathfrak{F}$.

1.4. As a general reference we shall use Reidemeister [1] for all facts concerning free groups that are used in the sequel without detailed justification. However, for the convenience of the reader and also to maintain the continuity of the presentation, we shall discuss briefly certain simple facts and we shall indicate proofs in some cases that may have occurred in previous literature.

\section{Preliminaries.}


2.1. $\mathfrak{F}$ will denote the free group over the free generators $S_{1}, \cdots$, $S_{j}, \cdots$, whose number (finite or countably infinite) will be termed the rank $\rho(\mathfrak{F})$ of $\mathfrak{F}$. As a generic notation for a generator $S_{1}, \cdots, S_{j}, \cdots$, we shall use the letter $S$. The Greek letters $\epsilon, \eta, \sigma$ will have the values \pm 1 and will be used as exponents. A word is then a (finite) expression of the form

$$
S_{j_{1}}^{\epsilon_{1}} \cdots S_{j_{r}}^{\epsilon_{r}}
$$

A word is reducible if there occurs in it at least one pair of adjacent factors $S^{\epsilon} S^{-\epsilon}$. The removal of such a pair and the inverse operation of inserting such a pair will be termed elementary modifications. A word which is not reducible is termed reduced. A formula of the form

$$
S_{j_{1}}^{\epsilon_{1}} \cdots S_{j_{r}}^{\epsilon_{r}} \equiv S_{k_{1}}^{\eta_{1}} \cdots S_{k_{t}}^{\eta_{t}}
$$

will mean that the two words involved are identical (that is, $r=t, j_{1}=k_{1}$, $\left.j_{2}=k_{2}, \cdots, \epsilon_{1}=\eta_{1}, \epsilon_{2}=\eta_{2}, \cdots\right)$. The symbol $W_{0}$ will denote the empty word. In contradistinction with (1), a formula of the form

$$
S_{j_{1}}^{\epsilon_{1}} \cdots S_{j_{r}}^{\epsilon_{r}}=S_{k_{1}}^{\eta_{1}} \cdots S_{k_{t}}^{\eta_{t}}
$$

will mean that the two words are expressions for the same element $f$ of $\mathfrak{F}$ (that is, either word can be derived from the other one by a finite number of elementary modifications). If $f_{1}, \cdots, f_{k}$ are elements of $\mathfrak{F}$, then the expression $f_{1} \cdots f_{k}$ will be said to be reduced as written if on replacing each $f_{i}, i=1$, $\cdots, k$, by its (univocally determined) reduced expression in terms of the generators $S$, we obtain a reduced word.

2.2. Given in $\mathfrak{F}$ a Schreier system $g_{1}, \cdots, g_{i}, \cdots$ (see 1.1), we shall use $G$ as a generic notation for an element of the sequence $g_{1}, \cdots, g_{i}, \cdots$ It will be understood that $G$ is always written in reduced form, and that $G^{-1}$ denotes the formal inverse of $G$. Thus $G^{-1}$ is also in reduced form. $H$ will be a generic notation for an element of $\mathfrak{F}$ that can be written in the form GSe, $\epsilon= \pm 1$. For example, in the free group over two free generators $a, b$ we have the Schreier system $1, a, a b$, and $a$ itself is then an $H$ since $a=1 \cdot a=(a b) b^{-1}$. This example shows also that an element $H$ may admit of several representations of the form $G S^{\epsilon}$.

2.3. Given in $\mathfrak{F}$ a Schreier system $g_{1}, \cdots, g_{j}, \cdots$, a function $\phi(H)$, defined for every element $H \in \mathfrak{F}$ (see 2.2), will be termed admissible with respect to the given Schreier system if the following conditions hold.

(a) For every $H \in \mathfrak{F}, \phi(H)$ is equal to a $G$.

(b) If $H$ is equal to some $G$, say $H=g_{i}$, then $\phi(H)=g_{i}$.

(c) For every choice of $G, S, \epsilon$ we have $\phi\left[\phi\left(G S^{\epsilon}\right) S^{-\epsilon}\right]=G$.

We noted above that an element $H$ may admit of several representations of the form $G S^{\epsilon}$. However, our notation $\phi(H)$ is meant to imply that $\phi(H)$ de- 
pends only upon $H$ itself and not upon the particular choice of the representation $G S^{\epsilon}$.

Given a Schreier system and an admissible function $\phi(H)$, the letter $V$ will be used as a generic notation for an element of $\mathfrak{F}$ that can be written in the form

$$
V=G S^{\epsilon}\left[\phi\left(G S^{\epsilon}\right)\right]^{-1} .
$$

In connection with the expression on the right in (1), we make the following agreement. Since $\phi\left(G S^{\epsilon}\right)$ is equal to some $g_{i}$ (see (a) above), we agree that $\phi\left(G S^{\epsilon}\right)$ is always written in reduced form, and that $\left[\phi\left(G S^{\epsilon}\right)\right]^{-1}$ is always written as the formal inverse of $\phi\left(G S^{\epsilon}\right)$.

The case where $\epsilon=+1$ in (1) is of importance. We shall use $U$ as a generic notation for an element of $\mathfrak{F}$ that can be written in the form

$$
U=G S[\phi(G S)]^{-1} .
$$

Thus a $U$ is merely a special $V$, and the conventions agreed upon for $V$ apply to $U$.

Let us note that there is no a priori assumption made that a $V$ can be written in the form (1) in a unique manner. This point will be studied later on.

2.4. To illustrate and to motivate the preceding definitions, let us consider a subgroup $\mathfrak{U}$ of $\mathfrak{F}$ and an associated Schreier system $g_{1}, \cdots, g_{i}, \cdots$ (see 1.2). If $f_{1}, f_{2}$ are two elements of $\mathfrak{F}$, and if $f_{1}, f_{2}$ are in the same left coset relative to $\mathfrak{U}$, then we shall write $f_{1} \sim f_{2}(\bmod \mathfrak{U})$, or simply $f_{1} \sim f_{2}$ if there is no danger of ambiguity. For each element $f$ of $\mathfrak{F}$, we have then a unique $G$ such that $f \sim G$. The symbol $\Psi(f)$ will then denote this unique $G$. Thus an equation of the form $\Psi(f)=G$ is equivalent to the relation ' $f \sim G$. Clearly, the function $\Psi(f)$ is univocally determined by the subgroup $\mathfrak{U}$ and the associated Schreier system $g_{1}, \cdots, g_{i}, \cdots$. The following statements hold.

(a) $\Psi(f)$ is always equal to some $G$. This is a direct consequence of the definition.

(b) $\Psi(G)=G$, also as an obvious consequence of the definition.

(c) $\Psi\left[\Psi\left(G S^{\epsilon}\right) S^{-\epsilon}\right]=G$. Indeed, by definition, $\Psi\left(G S^{\epsilon}\right) \sim G S^{\epsilon}$ and hence $\Psi\left(G S^{\epsilon}\right) S^{-\epsilon} \sim G$. Thus the asserted relation is obvious.

Now let us put $\phi(H)=\Psi(H)$. In view of the preceding remarks, this $\phi(H)$ is admissible for the given Schreier system, in the sense of 2.3.

2.5. Continuation. The particular $\phi(H)$ just considered will be said to be associated with the subgroup $\mathfrak{U}$ and the Schreier system $g_{1}, \cdots, g_{i}, \cdots$ In this manner, we see that if we have a subgroup $\mathfrak{U}$ and an associated Schreier system $g_{1}, \cdots, g_{i}, \cdots$, then the associated function $\phi(H)$ is admissible for the Schreier system $g_{1}, \cdots, g_{i}, \cdots$.

2.6. In view of the preceding situation there arise the following problems.

(a) Given a Schreier system $g_{1}, \cdots, g_{i}, \cdots$, determine all subgroups $\mathfrak{U}$ that are associated with it. 
(b) Given a Schreier system $g_{1}, \cdots, g_{i}, \cdots$, determine all admissible functions $\phi(H)$.

(c) Given a Schreier system $g_{1}, \cdots, g_{i}, \cdots$, and an admissible function $\phi(H)$, determine all subgroups $\mathfrak{U}$ such that $\phi(H)$ is associated with $\mathfrak{U}$ and the given Schreier system, in the sense of 2.5 .

2.7. In dealing with the preceding issues, we shall use a certain condition, termed condition (C), that we shall state presently. Given a Schreier system $g_{1}, \cdots, g_{j}, \cdots$, let us consider for each generator $S_{j}$ of $\mathfrak{F}$ the sequences

$$
\begin{gathered}
g_{1} S_{j}, g_{2} S_{j}, \cdots, g_{i} S_{j}, \cdots, \\
g_{1} S_{j}^{-1}, g_{2} S_{j}^{-1}, \cdots, g_{i} S_{j}^{-1}, \cdots .
\end{gathered}
$$

Let us denote by $I_{j}^{+}$the class of those subscripts $i$ for which the product $g_{i} S_{j}$ is equal to some $G$. Thus $i \in I_{j}^{+}$if and only if there exists a subscript $k$ such that $g_{i} S_{j}=g_{k}$. Similarly, we denote by $I_{\bar{j}}$ the class of those subscripts $i$ for which the product $g_{i} S_{j}^{-1}$ is equal to some $G$. Let $N_{j}^{+}, M_{j}^{+}$be the number (possibly infinite) of the subscripts $i$ satisfying the relations $i \in I_{j}^{+}, i \notin I_{j}^{+}$respectively. Similarly, let $N_{\bar{j}}, M_{j}^{-1}$ be the number of those subscripts $i$ that satisfy the relations $i \in I_{\bar{j}}, i \notin I_{\bar{j}}$. respectively. Finally, let $N$ be the number (possibly infinite) of the elements of the given Schreier system $g_{1}, \cdots, g_{i}, \cdots$ Clearly

$$
\begin{aligned}
N_{j}^{+}+M_{j}^{+} & =N=N_{\bar{j}}+M_{\bar{j}}, \\
N_{j}^{+} & =N_{\bar{j}} .
\end{aligned}
$$

Hence we have also

$$
M_{j}^{+}=M_{\bar{j}}^{-} \quad \text {.if } N<+\infty .
$$

On the other hand, simple examples show that the relation $M_{j}^{+}=M_{\bar{j}}^{-}$may fail to hold if $N=+\infty$ (example: choose $\mathfrak{F}$ as the free cyclic group with generator $S$, and consider the Schreier system $\left.1, S, S^{2}, \cdots, S^{n}, \cdots\right)$. We state now the following definition.

Condition (C). A Schreier system $g_{1}, \cdots, g_{i}, \cdots$ is said to satisfy condition (C) if and only if $M_{j}^{+}=M_{j}^{-}$for every generator $S_{j}$ of $\mathfrak{F}$.

According to (3), every finite Schreier system satisfies the condition (C).

2.8. Suppose now that for a given Schreier system $g_{1}, \cdots, g_{i}, \cdots$ there exists an admissible function $\phi(H)$, in the sense of 2.3. We assert that the Schreier system satisfies the condition (C). In other words, condition (C) is necessary for the existence of an admissible function $\phi(H)$ (the sufficiency of the condition will be established in \$4).

Proof. To show that $M_{j}^{+}=M_{\bar{j}}^{-}$(see 2.7), we first establish the inequality

$$
M_{j}^{+} \leqq M_{\bar{j}} \text {. }
$$

If $M_{j}^{+}=0$, then (1) is obvious. So we assume that $M_{j}^{+}>0$. Let $m$ be any in- 
teger such that

$$
0<m \leqq M_{j}^{+} .
$$

If $M_{j}^{+}<+\infty$, then we can choose $m=M_{j}^{+}$, but the following argument is designed to cover the case $M_{j}^{+}=+\infty$ also. By the definition of $M_{j}^{+},(2)$ implies the existence of distinct subscripts $i_{1}, \cdots, i_{m}$ such that (see 2.7)

$$
i_{r} \notin I_{j}^{+}, \quad r=1, \cdots, m .
$$

Let us put (cf. 2.3)

$$
\phi\left(g_{i}, S_{j}\right)=g_{k_{r}}, \quad r=1, \cdots, m .
$$

We assert that (see 2.7)

$$
k_{r} \notin I_{\bar{j}}, \quad \quad r=1, \cdots, m .
$$

Indeed, suppose that for example $k_{1} \in I_{j}^{-}$. Then $g_{k_{1}} S_{j}^{-1}$ is equal to some $G$, say $g_{k_{1}} S_{j}^{-1}=g_{\imath}$. By 2.3 (b) we have then

$$
\phi\left(g_{k 1} S_{j}^{-1}\right)=g_{t} .
$$

By 2.3 (c) it follows that (cf. (4))

$$
g_{t}=\phi\left[\phi\left(g_{i_{1}} S_{j}\right) S_{j}^{-1}\right]=g_{i_{1}} .
$$

Hence $g_{i_{1}}=g_{k_{1}} S_{j}^{-1}, g_{i_{1}} S_{j}=g_{k_{1}}$, and hence $i_{1} \in I_{j}^{+}$in contradiction with (3). Thus (5) is verified. Since $k_{1}, \cdots, k_{m}$ are clearly distinct integers, it follows that $M_{j}^{-} \leqq m$. Since this holds for every $m$ satisfying (2), the inequality (1) follows. The complementary inequality $M_{j}^{-} \leqq M_{j}^{+}$is established in a similar manner.

2.9. Suppose now that for a given Schreier system $g_{1}, \cdots, g_{i}, \cdots$ there exists an associated subgroup $\mathfrak{U}$ in the sense of 1.2. We assert that the Schreier system satisfies then the condition (C) of 2.7. In other words, condition (C) is necessary for the existence of an associated subgroup (the sufficiency of the condition will be established in 5.9).

Proof. By 2.4 the existence of an associated subgroup implies the existence of an admissible function $\phi(H)$, which in turn implies condition $(\mathrm{C})$ by 2.8 .

3. Properties of the admissible function $\phi(H)$.

3.1. Let $g_{1}, \cdots, g_{i}, \cdots$ be a Schreier system in $\mathfrak{F}$, and let $\phi(H)$ be an admissible function in the sense of 2.3 (the existence of such a function will be discussed later on). To simplify our statements, we introduce three matrices to be termed the $G S^{\epsilon}$ matrix, the $\phi\left(G S^{\epsilon}\right)$ matrix, and the $G S^{\epsilon}\left[\phi\left(G S^{\epsilon}\right)\right]^{-1}$ matrix respectively. The columns of the $G S^{\bullet}$ matrix (see figure) are headed by $S_{1}, S_{1}^{-1}, S_{2}, S_{2}^{-1}, \ldots$ The general column will be referred to as the $S^{\epsilon}$ column. The rows are headed by $g_{1}=1, g_{2}, \cdots, g_{i}, \cdots$. The general row will be termed the $G$ row. At the intersection of the $i$ th row and the $j$ th column we have the element $l_{i j}$ defined by the agreement: 


$$
l_{i j}=\left\{\begin{array}{l}
g_{i} S_{k} \\
g_{i} S_{k}^{-1}
\end{array}\right.
$$$$
\text { if } j=2 k-1 \text {, }
$$$$
\text { if } j=2 k \text {, }
$$

where $k=1,2, \cdots$. Thus the general element of this matrix is of the form $G S^{\epsilon}$, where

\begin{tabular}{|c|c|c|c|c|c|}
\hline$G S^{S^{\prime}}$ & $S_{1}$ & $S_{1}^{-1}$ & $S_{2}$ & $S_{2}^{-1}$ & $\ldots$ \\
\hline$g_{1}$ & $l_{11}$ & $l_{12}$ & $l_{13}$ & $l_{14}$ & $\ldots$ \\
\hline$g_{2}$ & $l_{21}$ & $l_{22}$ & $l_{23}$ & $l_{24}$ & $\ldots$ \\
\hline$g_{3}$ & $l_{31}$ & $l_{32}$ & $l_{33}$ & $l_{34}$ & $\ldots$ \\
\hline$\vdots$ & $\vdots$ & $\vdots$ & $\vdots$ & $\vdots$ & \\
\hline
\end{tabular}

$G$ heads the row and $S^{\epsilon}$ heads the column which intersect in the element considered. Hence each $l_{i j}$ is an $H$ (see 2.2), and hence $\phi\left(l_{i j}\right)$ is meaningful. The general element $\lambda_{i j}$ of the matrix $\phi\left(G S^{\epsilon}\right)$ is now defined as $\lambda_{i j}=\phi\left(l_{i j}\right)$. Thus each $\lambda_{i j}$ is of the form $\phi\left(G S^{e}\right)$. Hence each $\lambda_{i j}$ is a $G$ (see 2.3). Finally, we define the general element $\Lambda_{i j}$ of the matrix $G S^{e}\left[\phi\left(G S^{e}\right)\right]^{-1}$ by the formula $\Lambda_{i j}=l_{i j} \lambda_{i j}^{-1}$. Thus each $\Lambda_{i j}$ is of the form $G S^{e}\left[\phi\left(G S^{e}\right)\right]^{-1}$. Hence each $\Lambda_{i j}$ is a $V$ (see 2.3). In writing the elements of these three matrices, we agree to observe the conventions made in 2.2, 2.3.

3.2. If a certain $l_{i j}$ is a $G$, say $l_{i j}=g_{k}$, then $\lambda_{i j}=g_{k}$. This is a direct consequence of the property 2.3 (b). Furthermore, we assert that each column of the $\phi\left(G S^{c}\right)$ matrix is a permutation (rearrangement) of the given Schreier system $g_{1}, \cdots, g_{i}, \cdots$. We make the proof in two steps.

(a) Consider in the $\phi\left(G S^{e}\right)$ matrix the column headed by $S^{e}$, and suppose that $\phi\left(g_{i} S^{c}\right)=\phi\left(g_{k} S^{c}\right)$ for a certain pair of integers $i \neq k$. Then $\phi\left(g_{i} S^{e}\right) S^{-\bullet}$ $=\phi\left(g_{k} S^{\epsilon}\right) S^{-}$, and hence by the property 2.3 (c) it follows that

$$
g_{i}=\phi\left[\phi\left(g_{i} S^{\epsilon}\right) S^{-\epsilon}\right]=\phi\left[\phi\left(g_{k} S^{\epsilon}\right) S^{-\epsilon}\right]=g_{k},
$$

and this is a contradiction since $g_{i} \neq g_{h}$ for $i \neq k$. Thus no $G$ occurs twice in the column headed by $S^{\epsilon}$.

(b) We have to show yet that each $G$ actually occurs in the column headed by $S^{\epsilon}$. So let us take a $G=g_{i}$. Then $\phi\left(g_{i} S^{-\epsilon}\right)$ is equal to some $G$ again, say $\phi\left(g_{i} S^{-\epsilon}\right)=g_{k}$. Then by the property 2.3 (c) we have 


$$
\phi\left(g_{k} S^{\epsilon}\right)=\phi\left[\phi\left(g_{i} S^{-\epsilon}\right) S^{\epsilon}\right]=g_{i} .
$$

Thus $g_{i}$ occurs in the column headed by $S^{\epsilon}$, namely at the intersection with the row headed by $g_{k}$.

3.3. In the $\phi\left(G S^{\epsilon}\right)$ matrix, the column headed by $S_{j}^{-1}$ can be derived from the column headed by $S_{j}$ as follows. Consider the element $\phi\left(g_{i} S_{j}^{-1}\right)$ at the intersection of the column headed by $S_{j}^{-1}$ with the row headed by $g_{i}$. Now in the column headed by $S_{j}$ this $g_{i}$ occurs exactly once (see 3.2 ), say in the row headed by $g_{k}$. Then $\phi\left(g_{k} S_{j}\right)=g_{i}$, and hence by the property 2.3 (c) it follows that

$$
\phi\left(g_{i} S_{j}^{-1}\right)=\phi\left[\phi\left(g_{k} S_{j}\right) S_{j}^{-1}\right]=g_{k} .
$$

3.4. We go on to consider the matrix $\Lambda_{i j}$. As noted in 3.1, each $\Lambda_{i j}$ is a $V$. Consider any

$$
V=G S^{\epsilon}\left[\phi\left(G S^{\epsilon}\right)\right]^{-1} \text {. }
$$

We assert that $V=1$ if and only if $G S^{\epsilon}$ is equal to some $g_{i}$. Indeed, if $V=1$, then $G S^{\epsilon}=\phi\left(G S^{\epsilon}\right)=$ some $g_{i}$ by the property 2.3 (a). Suppose, conversely, that $G S^{\epsilon}=g_{i}$. By the property 2.3 (b) we have then $\phi\left(G S^{\epsilon}\right)=g_{i}=G S^{\epsilon}$, and hence $V=1$.

3.5. If $G S^{\epsilon}$ is reducible as written (see 2.1), then $G S^{\epsilon}=$ some $g_{i}$.

Proof. Since $G$ itself is in reduced form, the reduction in $G S^{\epsilon}$ takes place between $G$ and $S^{\epsilon}$. Thus surely $G \neq 1$, and since $G$ is an element of a Schreier system, it follows that $G$ is of the form $G=g_{i} S^{-\epsilon}$ (cf. 1.1). Hence $G S^{\epsilon}=g_{i}$.

3.6. If $S^{\epsilon}\left[\phi\left(G S^{\epsilon}\right)\right]^{-1}$ is reducible as written, then $\phi\left(G S^{\epsilon}\right) S^{-\epsilon}=$ some $g_{i}$.

Proof. The assumption implies that $\phi\left(G S^{\epsilon}\right) S^{-\epsilon}$ is also reducible as written. But $\phi\left(G S^{\epsilon}\right)=$ some $g_{k}$, and hence from 3.5 it follows that $\phi\left(G S^{\epsilon}\right) S^{-\bullet}$ $=$ some $g_{i}$.

3.7. If $V=G S^{\epsilon}\left[\phi\left(G S^{\epsilon}\right)\right]^{-1}$, then on setting $\phi\left(G S^{\epsilon}\right)=G^{*}$ (see 2.3 (a)), we have the formula

$$
V^{-1}=G^{*} S^{-\epsilon}\left[\phi\left(G^{*} S^{-\epsilon}\right)\right]^{-1} .
$$

In other words, the inverse of a $V$ is also a $V$ (cf. 2.3).

Proof. By 2.3 (c) we have

$$
\phi\left(G^{*} S^{-\epsilon}\right)=\phi\left[\phi\left(G S^{\epsilon}\right) S^{-\epsilon}\right]=G,
$$

and the assertion follows.

3.8. If $1 \neq V=G S^{e}\left[\phi\left(G S^{e}\right)\right]^{-1}$, then $V$ is reduced as written (see 2.1).

Proof. Since $G$ and $\left[\phi\left(G S^{e}\right)\right]^{-1}$ are written in reduced form, it is sufficient to show that $G S^{\epsilon}$ and $S^{\epsilon}\left[\phi\left(G S^{\epsilon}\right)\right]^{-1}$ are reduced as written.

(a) Assume that $G S^{e}$ is reducible as written. Then $G S^{e}=$ some $g_{i}$ by 3.5 , and hence by 3.4 we should have $V=1$, a contradiction.

(b) Assume that $S^{\epsilon}\left[\phi\left(G S^{\epsilon}\right)\right]^{-1}$ is reducible as written. On setting $\phi\left(G S^{\epsilon}\right)$ 
$=G^{*}$, we have then by 3.7

$$
V^{-1}=G^{*} S^{-\epsilon}\left[\phi\left(G^{*} S^{-\epsilon}\right)\right]^{-1} .
$$

By 3.6, $\phi\left(G S^{e}\right) S^{-\epsilon}=G^{*} S^{-\epsilon}=$ some $g_{i}$. Applying 3.4 to $V^{-1}$ (cf. 3.7), it follows that $V^{-1}=1$ and hence $V=1$, a contradiction.

3.9. We are now in a position to verify a fundamental property of the matrix $G S^{\epsilon}\left[\phi\left(G S^{\epsilon}\right)\right]^{-1}$. Given

$$
V_{1}=g_{i_{1}} S_{j_{1}}^{\epsilon_{1}}\left[\phi\left(g_{i_{1}} S_{j_{1}}^{\epsilon_{1}}\right)\right]^{-1}, \quad V_{2}=g_{i_{2}} S_{j_{2}}^{\epsilon_{2}}\left[\phi\left(g_{i_{2}} S_{j_{2}}^{\epsilon_{2}}\right)\right]^{-1},
$$

suppose that $1 \neq V_{1}=V_{2}$. Then we assert that $i_{1}=i_{2}, j_{1}=j_{2}, \epsilon_{1}=\epsilon_{2}$. In other words, a $V \neq 1$ can be written in the form $G S^{\epsilon}\left[\phi\left(G S^{\epsilon}\right)\right]^{-1}$ in just one way.

Proof. By 3.8 we know that $V_{1}$ and $V_{2}$ are reduced as written. Hence the assumption $V_{1}=V_{2}$ implies that

$$
g_{i_{1}} S_{j_{1}}^{\epsilon_{1}}\left[\phi\left(g_{i_{1}} S_{j_{1}}^{\epsilon_{1}}\right)\right]^{-1} \equiv g_{i_{2}} S_{j_{2}}^{\epsilon_{2}}\left[\phi\left(g_{i_{2}} S_{j_{2}}^{\epsilon_{2}}\right)\right]^{-1},
$$

where the symbol " $\equiv$ " means that the two sides of (1) are identical words in terms of the generators $S$ (cf. 2.2, 2.3).

Case 1. $g_{i_{1}}, g_{i_{2}}$ are of the same length (as reduced words). Then (1) implies that $g_{i_{1}}=g_{i_{2}}, S_{j_{1}}^{\epsilon_{1}}=S_{j_{2}}^{\epsilon_{2}}$, and hence $i_{1}=i_{2}, j_{1}=j_{2}, \epsilon_{1}=\epsilon_{2}$.

Case 2. $g_{i_{1}}, g_{i_{2}}$ are of different lengths, say $g_{i_{2}}$ is longer than $g_{i_{1}}$. Then (1) implies that $g_{i_{1}} S_{j_{1}}^{\epsilon_{1}}$ is identical with a beginning section of $g_{i_{2}}$ (which may coincide with $g_{i_{2}}$ itself). Thus (see 1.1) $g_{i_{1}} S_{j_{1}}^{\varphi_{1}}$ is equal to some $G$, and hence by 3.4 it follows that $V_{1}=1$, a contradiction. Thus Case 2 cannot arise, and the proof is complete.

3.10. In view of the preceding result, for each $V \neq 1$ we have a unique expression of the form $G S^{\epsilon}\left[\phi\left(G S^{\epsilon}\right)\right]^{-1}$, and thus in particular the factor $S^{\epsilon}$ is univocally determined by $V$ if $V \neq 1$. This univocally determined factor will be termed the significant factor of $V \neq 1$. By 3.7 we have then the statement:

If $S^{\epsilon}$ is the significant factor of $V \neq 1$, then the significant factor of $V^{-1}$ is $S^{-\epsilon}$.

3.11. Suppose that $V_{1} \neq 1, V_{2} \neq 1, V_{2} \neq V_{1}^{-}$. Then $V_{1}, V_{2}$ have by 3.9 unique expressions of the form

$$
V_{1}=g_{i_{1}} S_{j_{1}}^{\epsilon_{1}}\left[\phi\left(g_{i_{1}} S_{j_{1}}^{\epsilon_{1}}\right)\right]^{-1}, \quad V_{2}=g_{i_{2}} S_{j_{2}}^{\epsilon_{2}}\left[\phi\left(g_{i_{2}} S_{j_{2}}^{\epsilon_{2}}\right)\right]^{-1} \text {. }
$$

Let us consider the expression

$$
g_{i_{1}} S_{j_{1}}^{\epsilon_{1}}\left[\phi\left(g_{i_{1}} S_{j_{1}}^{\epsilon_{1}}\right)\right]^{-1} g_{i_{2}} S_{j_{2}}^{\epsilon_{2}}\left[\phi\left(g_{i_{2}} S_{j_{2}}^{\epsilon_{2}}\right)\right]^{-1} .
$$

Since $V_{1}, V_{2}$ are both reduced as written by $3: 8$, the expression (1) is either reduced as written or else the reduction spreads from between $\left[\phi\left(g_{i_{1}} S_{\gamma_{1}}^{q_{1}}\right)\right]^{-1}$ and $g_{i_{2}}$. Assuming that (1) is not reduced as written, we assert that the significant factors $S_{j_{1}}^{\epsilon_{1}}, S_{j_{2}}^{\epsilon_{2}}$ of $V_{1}, V_{2}$ cannot be reached by the possible reductions. Let us deny this assertion.

Case 1. $\phi\left(g_{i_{1}} S_{j_{1}}^{e_{1}}\right)$ and $g_{i_{2}}$ are of the same length (written in reduced form 
as agreed in $2.2,2.3)$. Then the reductions reach $S_{j_{1}}^{\epsilon_{1}}, S_{j_{2}}^{\epsilon_{2}}$ simultaneously, and it follows that

$$
g_{i_{2}}=\phi\left(g_{i_{1}} S_{j_{1}}^{\epsilon_{1}}\right), \quad S_{j_{2}}^{\epsilon_{2} \cdots}=S_{j_{1}}^{-\epsilon_{1}}
$$

By 2.3 (c) it follows that

$$
\phi\left(g_{i_{2}} S_{j_{2}}^{\epsilon_{2}}\right)=\phi\left[\phi\left(g_{i_{1}} S_{j_{1}}^{\epsilon_{1}}\right) S_{j_{2}}^{\epsilon_{2}}\right]=\phi\left[\phi\left(g_{i_{1}} S_{j_{1}}^{\epsilon_{1}}\right) S_{j_{1}}^{-\epsilon_{1}}\right]=g_{i_{1}},
$$

and consequently $V_{1}=V_{2}^{-1}$, a contradiction.

Case 2. $g_{i_{2}}$ is longer than $\phi\left(g_{i_{1}} S_{j_{1}}^{\epsilon_{1}}\right)$ (in using the reduced forms). Then the reduction reaches $S_{j_{1}}^{\epsilon_{1}}$ first, and thus $\phi\left(g_{i_{1}} S_{j_{1}}^{\epsilon_{1}}\right) S_{j_{1}}^{-\epsilon_{1}}$ is equal to a beginning section of $g_{i_{2}}$ (which may coincide with $g_{i_{2}}$ itself). Thus $\phi\left(g_{i_{1}} S_{j_{1}}^{\epsilon_{1}}\right) S_{j_{1}}^{-\epsilon_{1}}$ is equal to some $G$, say $g_{k}$, and from 2.3 (c), 2.3 (b) it follows that

$$
g_{k}=\phi\left[\phi\left(g_{i_{1}} S_{j_{1}}^{\epsilon_{1}}\right) S_{j_{1}}^{-\epsilon_{1}}\right]=g_{i_{1}} .
$$

Consequently

$$
\phi\left(g_{i_{1}} S_{j_{1}}^{\epsilon_{1}}\right) S_{j_{1}}^{-\epsilon_{1}}=g_{i_{1}},
$$

and hence $V_{1}=1$, a contradiction.

Case 3. $\phi\left(g_{i_{1}} S_{j_{1}}^{\epsilon_{1}}\right)$ is longer than $g_{i_{2}}$ (in using the reduced form). Then the reduction reaches $S_{j_{2}}^{\epsilon_{2}}$ first, and thus $g_{i_{2}} S_{j_{2}}^{\epsilon_{2}}$ is equal to a beginning section of $\phi\left(g_{i_{1}} S_{j_{1}}^{\epsilon_{1}}\right)$. This latter quantity being a $G$, it follows that $g_{i_{2}} S_{j_{2}}^{\epsilon_{2}}$ is also a $G$, and hence by 3.4 it follows that $V_{2}=1$, a contradiction.

3.12. Now consider a product $V_{1} V_{2} \cdots V_{k}$, such that $V_{i} \neq 1, i=1$, $\cdots, k$, and $V_{j} \neq V_{j+1}^{-1}, j=1, \cdots, k-1$ (but we do not exclude the case where $V_{i}=V_{j}$ for $i \neq j$ ). We assert that $V_{1} V_{2} \cdots V_{k} \neq 1$.

Proof. Let each $V_{j}$ be written in the form $G S^{\epsilon}\left[\phi\left(G S^{\epsilon}\right)\right]^{-1}$. From 3.8, 3.11 we infer that if reductions are possible at all in the product $V_{1} V_{2} \cdots V_{k}$, then the reductions spread from between adjacent factors $V$ and never reach the significant factors. Thus on completing all possible reductions, we obtain a nonempty reduced word, and hence $V_{1} V_{2} \cdots V_{k} \neq 1$.

3.13. Now let $U_{1}, U_{2}, \cdots$ be the (finite or countably infinite) sequence of the elements $U \neq 1$ (see 2.3). It is understood that each $U \neq 1$ is listed just once. In other words, $U_{i}=U_{j}$ if and only if $i=j$. We assert that an equation of the form $U_{i_{1}}^{\epsilon_{1}}=U_{i_{2}}^{\epsilon_{2}}$ holds if and only if $i_{1}=i_{2}, \epsilon_{1}=\epsilon_{2}$. Indeed, let $S_{k_{1}}, S_{k_{2}}$ be the significant factors of $U_{i_{1}}, U_{i_{2}}$. In view of 3.10, the significant factors of $U_{i_{1}}^{\epsilon_{1}}, U_{\mathfrak{t}_{2}}^{\epsilon_{2}}$ are $S_{\mathbf{k}_{1}}^{\epsilon_{1}}, S_{\boldsymbol{k}_{2}}^{\epsilon_{2}}$ respectively. By 3.10 it follows that $S_{\boldsymbol{k}_{1}}^{\epsilon_{1}}=S_{\boldsymbol{k}_{2}}^{\epsilon_{2}}$, and hence $k_{1}=k_{2}, \epsilon_{1}=\epsilon_{2}$. It follows that $U_{i_{1}}^{\epsilon_{1}}=U_{i_{2}}^{\epsilon_{1}}$ and hence $U_{i_{1}}=U_{i_{2}}$. Thus $i_{1}=i_{2}$, and the proof is complete.

3.14. Now consider any product $U_{i_{1}}^{\epsilon_{1}} \cdots U_{i_{r}}^{\epsilon_{r}}$, such that for no $k=1,2$, $\cdots, r-1$ do the relations $i_{k}=i_{k+1}, \epsilon_{k}=-\epsilon_{k+1}$ hold simultaneously. We assert that

$$
U_{i_{1}}^{\epsilon_{1}} \cdots U_{i_{r}}^{\epsilon_{r}} \neq 1
$$


Let us deny this assertion. Put $U_{i_{k}}^{\epsilon_{k}}=V_{k}, k=1, \cdots, r$ (cf. 3.7, 2.3). We would have then the relation $V_{1} \cdots V_{r}=1$. By 3.12 it follows that $V_{j}=V_{j+1}^{-1}$ for at least one integer $j=1, \cdots, r-1$. In other words, we would have a relation

$$
U_{i_{j}}^{\epsilon_{j}}=U_{i_{j+1}}^{-\epsilon_{j+1}} .
$$

By 3.13 it follows that $i_{j}=i_{j+1}, \epsilon_{j}=-\epsilon_{j+1}$, in contradiction with our assumptions.

3.15. Now let us denote by $\mathfrak{U}$ the subgroup of $\mathfrak{F}$ generated by the elements $U_{1}, U_{2}, \cdots$ (that is, $\mathfrak{U}$ is the smallest subgroup of $\mathfrak{F}$ that contains $\left.U_{1}, U_{2}, \cdots\right)$. This subgroup $\mathfrak{U}$ is univocally determined by the given Schreier system $\{G\}=\left(g_{1}, \cdots, g_{i}, \cdots\right)$ and by the admissible function $\phi(H)$, and will be denoted by $\mathfrak{U}[\{G\}, \phi(H)]$. Let us note that the Schreier system $g_{1}, \cdots, g_{i}, \cdots$ by itself does not determine a subgroup $\mathfrak{U}$, since the generators $U_{1}, U_{2}, \cdots$ depend upon the choice of an admissible function $\phi(H)$. In a subsequent chapter, we shall consider this point in more detail. For the moment, we merely note that the result in 3.14 means that $U_{1}, U_{2}, \cdots$ are free generators of $\mathfrak{u}[\{G\}, \phi(H)]$. In other words, the subgroup $\mathfrak{U}$ generated by a Schreier system and an admissible function $\phi(H)$ is always a free group, and the rank $\rho(\mathfrak{U})$ (see 2.1 ) is equal to the number of the elements $U \neq 1$. $\S 5$ is devoted to a more detailed study of the group $\mathfrak{U}$ generated in this manner.

4. Existence of admissible functions $\phi(H)$.

4.1. Let there be given in $\mathfrak{F}$ a Schreier system $g_{1}, \cdots, g_{i}, \cdots$ that satisfies condition (C) (see 2.7). We proceed to show the existence of at least one admissible function $\phi(H)$, and as a matter of fact we propose to determine all functions $\phi(H)$ that are admissible for the given Schreier system. In view of 3.1 , we have to define a matrix $\phi\left(G S^{\epsilon}\right)$ such that the properties (a), (b), (c) stated in 2.3 hold. We first give the explicit definition of the matrix

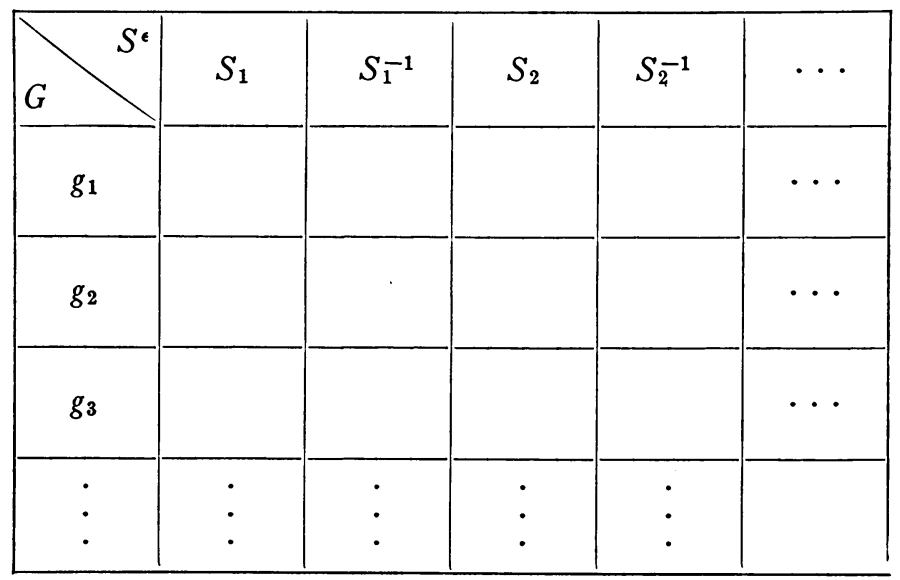


$\phi\left(G S^{e}\right)$, by giving explicit instructions for writing the element at the intersection of the column headed by $S^{\epsilon}$ with the row headed by $G=g_{i}$.

Step 1. Take the column headed by $S_{j}^{+1}$. Consider the intersection of this column with the row headed by a $G=g_{i}$. If $G S_{j}=$ some $g_{k}$, then we write this $g_{k}$ at the intersection considered. In other words, we define then $\phi\left(G S_{j}\right)=g_{k}$. Let $i, h$ be two different subscripts such that $g_{i} S_{j}^{+1}$ is equal to some $G=g_{k}$, and $g_{h} S_{j}^{+1}$ is equal to some $G=g_{t}$. We assert that $g_{k} \neq g_{t}$. Otherwise, we would have $g_{i} S_{j}=g_{h} S_{j}$ and hence $g_{i}=g_{h}$, a contradiction. Thus no two cells that were filled according to the preceding instruction contain the same $G$. After the above instruction has been followed for filling all those cells of the column $S_{j}^{+1}$ to which it is applicable, the remaining blank cells of this column are filled by the remaining elements $G$ subject only to the restriction that the column $S_{j}^{+1}$ should contain a permutation of the given Schreier system $g_{1}, \cdots, g_{i}, \cdots$. To show that this is indeed possible, we have to satisfy ourselves that the number of unexpended elements $G$ is equal to the number of the blank cells in the column $S_{j}^{+1}$ (these numbers may be infinite or zero). Now if a certain $G=g_{i}$ has not been used in the compulsory part of the process, then this is because for no subscript $k$ did we have the relation $g_{k} S_{j}=g_{i}$ or alternatively $g_{i} S_{j}^{-1}=g_{k}$. In other words, the number of unexpended elements $G$ is equal to the number $M_{j}^{-}$defined in 2.7. Next, if the cell at the intersection of the column $S_{j}^{+1}$ with the row headed by a certain $g_{i}$ is blank yet after the compulsory part of our process, then this is because $g_{i} S_{j}$ is not equal to any $G$. Thus the number of blank cells is equal to the number $M_{j}^{+}$defined in 2.7. Hence the number of unexpended elements $G$ is equal to the number of blank cells by virtue of condition (C) (see 2.7). Let us also note that our process for completing the column $S_{j}^{+1}$ is as general as possible in view of the remarks in 3.2 .

Step 2. After all columns headed by elements $S_{j}^{+1}$ have been completed, and hence $\phi\left(G S^{\epsilon}\right)$ has been defined for $\epsilon=+1$, we consider a column $S_{j}^{-1}$. Take the intersection of this column with the row headed by $g_{i}$. Since the column headed by $S_{j}^{+1}$ contains a permutation of the given Schreier system, we have precisely one $g_{k}$ such that $\phi\left(g_{k} S_{j}^{+1}\right)=g_{i}$. We put then $\phi\left(g_{i} S_{j}^{-1}\right)=g_{k}$. In this manner, the columns headed by elements of the form $S_{j}^{-1}$ are also completed. In view of 3.3 , it is clear that step 2 is altogether compulsory if our matrix $\phi\left(G S^{\epsilon}\right)$ is to have the desired properties.

4.2. Proceeding now to the verification of the properties (a), (b), (c) stated in 2.3, a first important point is whether the function $\phi(H)$ we just defined depends only upon $H$ and not upon the particular representation of $H$ in the form $G S^{e}$. To establish this point we have to show that whenever $g_{i} S_{r}^{\epsilon}=g_{k} S_{t}^{\eta}$, we have also $\phi\left(g_{i} S_{r}^{\epsilon}\right)=\phi\left(g_{k} S_{l}^{\eta}\right)$ according to our process of construction.

Case 1. $g_{i} S_{r}^{\epsilon}, g_{k} S_{t}^{\eta}$ are reduced as written. Then the assumption $g_{i} S_{r}^{\epsilon}=g_{k} S_{i}^{\eta}$ implies that $S_{r}^{\epsilon}=S_{t}^{\eta}, g_{i}=g_{k}$, and hence $r=t, \epsilon=\eta, i=k$. Hence $\phi\left(g_{i} S_{r}^{\epsilon}\right)=\phi\left(g_{k} S_{t}^{\eta}\right)$, since our instructions yielded a definite element for each cell of the matrix. 
Case 2. One at least of $g_{i} S_{r}^{e}, g_{k} S_{t}^{\eta}$ is reducible as written, say $g_{i} S_{r}^{e}$. Since $g_{i}$ itself is in reduced form, and since we are dealing with a Schreier system, $g_{i}$ must be of the form $g_{i}=g_{m} S_{r}^{-\epsilon}$. Thus

$$
g_{m}=g_{i} S_{r}^{\epsilon}=g_{k} S_{t}^{\eta} .
$$

By the definition of the matrix, we have therefore $\phi\left(g_{i} S_{r}^{e}\right)=g_{m}=\phi\left(g_{k} S_{t}^{\eta}\right)$.

4.3. The property 2.3 (a) obviously holds. To verify property 2.3 (b), let us assume that $g_{i} S_{j}^{\epsilon}=g_{k}$.

Case 1. $\epsilon=+1$. Then step 1 in 4.1 applies, and $\phi\left(g_{i} S_{j}^{+1}\right)=g_{k}$.

Case 2. $\epsilon=-1$. Then we are in the case considered in step 2 in 4.1. From $g_{i} S_{j}^{-1}=g_{k}, g_{k} S_{j}=g_{i}$ it follows, by Case 1 , that $\phi\left(g_{k} S_{j}\right)=g_{i}$, and hence $\phi\left(g_{i} S_{j}^{-1}\right)$ $=g_{k}$ by the rule stated in step 2 in 4.1 .

4.4. To verify the property 2.3 (c), let us consider $\phi\left[\phi\left(G S^{\epsilon}\right) S^{-\epsilon}\right]$.

Case 1. $\epsilon=+1$. Let $\phi(G S)=G^{*}$ (see 4.3). By step 2 in 4.1 we have then $\phi\left(G^{*} S^{-1}\right)=G$, and in view of 4.2 it follows that

$$
\phi\left[\phi(G S) S^{-1}\right]=\phi\left(G^{*} S^{-1}\right)=G .
$$

Case 2. $\epsilon=-1$. Put now $\phi\left(G S^{-1}\right)=G^{*}$. In view of step 2, 4.1, this means that $\phi\left(G^{*} S\right)=G$. Hence, in view of 4.2 it follows that

$$
\phi\left[\phi\left(G S^{-1}\right) S\right]=\phi\left(G^{*} S\right)=G .
$$

4.5. Thus our matrix actually defines an admissible function $\phi(H)$ for the given Schreier system. Beyond the mere existence of an admissible function $\phi(H)$, it is important to repeat the remark already made that our construction uses maximum freedom subject to the necessary conditions implied by the properties of admissible functions established in 3.2, 3.3. In other words, our construction yields all admissible functions for a given Schreier system. In view of 2.8 it follows that the condition $(\mathrm{C})$ of 2.7 is necessary and sufficient for the existence of an admissible function $\phi(H)$.

5. Study of the group $U[\{G\}, \phi(H)]$.

5.1. Given a Schreier system $g_{1}, \cdots, g_{i}, \cdots$ and an admissible function $\phi(H)$, let $\mathfrak{U}$ be the corresponding group $\mathfrak{U}[\{G\}, \phi(H)]$ (see 3.15). Consider, in the group $\mathfrak{F}$ (see 2.1 ), a word $S_{i_{1}}^{\epsilon_{1}} \cdots S_{i_{r}}^{\epsilon_{r}}$. To this word we apply a process, to be termed the $W$-process (see Reidemeister [1]), defined in the following manner. We write successively (cf. 2.1)

$$
W_{0} \equiv 1, W_{1} \equiv \phi\left(W_{0} S_{i_{1}}^{\epsilon_{1}}\right), W_{2} \equiv \phi\left(W_{1} S_{i_{2}}^{\epsilon_{2}}\right), \cdots, W_{r} \equiv \phi\left(W_{r-1} S_{i_{r}}^{\epsilon_{r}}\right) .
$$

The last term $W_{r}$ is the result of the $W$-process. Let us now consider the word

$$
S_{i_{1}}^{\epsilon_{1}} \cdots S_{i_{k}}^{\epsilon_{k}} S^{\epsilon} S^{-\epsilon} S_{i_{k+1}}^{\epsilon_{k+1}} \cdots S_{i_{r}}^{\epsilon_{r}}
$$


obtained by an elementary modification of the given word. For clarity, let us use $W_{0}^{*}, W_{1}^{*}, \cdots$ to denote the terms $W$ that arise in applying the $W$ process to the modified word (1). Then clearly

$$
W_{0}^{*} \equiv W_{0}, W_{1}^{*} \equiv W_{1}, \cdots, W_{k}^{*} \equiv W_{k},
$$

and hence

$$
\begin{aligned}
& W_{k+1}^{*} \equiv \phi\left(W_{k} S^{\epsilon}\right), \\
& W_{k+2}^{*} \equiv \phi\left(W_{k+1}^{*} S^{-\epsilon}\right) \equiv \phi\left[\phi\left(W_{k} S^{\epsilon}\right) S^{-\epsilon}\right] \equiv W_{k},
\end{aligned}
$$

by property 2.3 (c). Therefore we infer that

$$
\begin{aligned}
& W_{k+3}^{*} \equiv \phi\left(W_{k+2}^{*} S_{i_{k+1}}^{\epsilon_{k+1}}\right) \equiv \phi\left(W_{k} S_{i_{k+1}}^{\epsilon_{k+1}}\right) \equiv W_{k+1}, \\
& \cdot . \cdot . \cdot . \cdot . \cdot . \cdot . \cdot . \cdot . \cdot . ~ \\
& W_{r+2}^{*} \equiv \phi\left(W_{r+1}^{*} S_{i_{r}}^{\epsilon_{r}}\right) \equiv \phi\left(W_{r-1} S_{i_{r}}^{\epsilon_{r}}\right) \equiv W_{r} .
\end{aligned}
$$

It is thus established that an elementary modification of a word does not change the result of the $W$-process. Repeated application of this remark yields the insight that if two words are expressions for the same element of $\mathfrak{F}$, then the $W$-process yields the same result for both words. As a consequence, we can define in $\mathfrak{F}$ a function $\Phi(f)$ as follows: if $f=S_{\imath_{1}}^{\tau_{1}} \cdots S_{t_{r}}^{e_{r}}$, then $\Phi(f)$ is equal to the result of the $W$-process applied to the word $S_{t_{1}}^{\epsilon_{1}} \cdots S_{t_{r}}^{e_{r}}$ By the preceding remarks, this definition is legitimate since the result is independent of the particular expression chosen for $f$.

Thus $\Phi(f)$ is always a value of $\phi(H)$, and hence $\Phi(f)$ is always a $G$ (see 2.3). Let us note, in particular, that $\Phi(1)=1$. Indeed, if the $W$-process is applied to the empty word, then the result is $W_{0}=1$.

5.2. Let us write $f_{1} \sim f_{2}$ to state that the elements $f_{1}, f_{2}$ of $\mathfrak{F}$ are in the same left coset relative to the given subgroup $\mathfrak{U}$ (see 5.1). We assert that $f \sim \Phi(f)$ for every $f \in \mathfrak{F}$.

Proof. Let $f=S_{i_{1}}^{\epsilon_{1}} \cdots S_{t_{r}}^{\epsilon_{r}}$. Applying the $W$-process, we get

$$
W_{0} \equiv 1, W_{1} \equiv \phi\left(W_{0} S_{i_{1}}^{\epsilon_{1}}\right), \cdots, W_{r} \equiv \phi\left(W_{r-1} S_{i_{r}}^{\epsilon_{r}}\right) \equiv \Phi(f) .
$$

Consider now the product

$$
p \equiv W_{0} S_{i_{1}}^{\epsilon_{1}} W_{1}^{-1} \cdot W_{1} S_{i_{2}}^{\epsilon_{2}} W_{2}^{-1} \cdots W_{r-1} S_{i_{r}}^{\epsilon_{r}} W_{r}^{-1} .
$$

Since each $W_{j}, j=0,1, \cdots, r$, is a $G$, it follows that

$$
W_{j-1} S_{i_{j}}^{\epsilon_{j}} W_{j}^{-1} \equiv W_{j-1} S_{i_{j}}^{\epsilon_{j}}\left[\phi\left(W_{i_{j-1}} S_{i_{j}}^{\epsilon_{j}}\right)\right]^{-1}
$$

is equal to some $U^{\epsilon_{i}}$ (see $2.3,3.7$ ). Hence $p \in \mathfrak{U}$. On the other hand, clearly 


$$
p=f W_{r}^{-1}, \quad f=p W_{r}=p \Phi(f) .
$$

Since $p \in \mathfrak{U}$, this shows that $f \sim \Phi(f)$.

5.3. For each element $G$ of the given Schreier system, we have $\Phi(G)=G$.

Proof. Let $G=S_{i_{1}}^{\epsilon_{1}} \cdots S_{i_{r}}^{e_{r}}$, in reduced form. Recall that each beginning section of $G$ is then again a $G$. Hence, in view of the property 2.3 (b), the $W$ process yields successively

$$
W_{0} \equiv 1, W_{1} \equiv S_{i_{1}}^{\epsilon_{1}} W_{2} \equiv S_{i_{1}}^{\epsilon_{1}} S_{i_{2}}^{\epsilon_{2}}, \cdots, W_{r} \equiv S_{i_{1}}^{\epsilon_{1}} \cdots S_{i_{r}}^{\epsilon_{r}} \equiv G .
$$

Corollary. $\Phi[\Phi(f)]=\Phi(f)$. Indeed, $\Phi(f)$ is equal to some $G$.

5.4. For any two elements $f_{1}, f_{2}$ of $\mathfrak{F}$, we have

$$
\Phi\left(f_{1} f_{2}\right)=\Phi\left[\Phi\left(f_{1}\right) f_{2}\right] .
$$

Proof. Let

$$
f_{1}=S_{i_{1}}^{\epsilon_{1}} \cdots S_{i_{r}}^{\epsilon_{r}}, \Phi\left(f_{1}\right)=S_{k_{1}}^{\sigma_{1}} \cdots S_{k_{m}}^{\sigma_{m}}, f_{2}=S_{j_{1}}^{\eta_{1}} \cdots S_{j_{t}}^{\eta_{t}}
$$

In calculating $\Phi\left(f_{1} f_{2}\right)$, let the $W$-process yield successively $W_{0}, W_{1}, \cdots, W_{r+t}$, and let us denote by $W_{0}^{*}, W_{1}^{*}, \cdots, W_{m+t}^{*}$ the $W^{\prime}$ s obtained in applying the $W$-process to $\Phi\left(f_{1}\right) f_{2}$. In view of 5.3 we have then $W_{m}^{*}=\Phi\left(f_{1}\right)$, and clearly $W_{r}=\Phi\left(f_{1}\right)$. Hence

$$
W_{m+1}^{*} \equiv \phi\left(W_{m}^{*} S_{j_{1}}^{\eta_{1}}\right) \equiv \phi\left(W_{r} S_{j_{1}}^{\eta_{1}}\right) \equiv W_{r+1}
$$

and similarly $W_{m+2}^{*}=W_{r+2}, \cdots, W_{m+\imath}^{*}=W_{r+t}$.

5.5. $\Phi\left(U^{c}\right)=1$ (cf. 2.3).

In view of $3.7, U^{e}$ can be written in the form

$$
U^{\bullet}=G S^{\epsilon}\left[\phi\left(G S^{\epsilon}\right)\right]^{-1} \text {. }
$$

Let $r$ be the length of $G$ in reduced form. In view of 5.3 the $W$-process yields then

$$
W_{r} \equiv G, \quad W_{r+1} \equiv \phi\left(W_{r} S^{\epsilon}\right) \equiv \Phi\left(G S^{\epsilon}\right) .
$$

Hence, by 5.4 , we have

$$
\Phi\left(U^{\star}\right)=\Phi\left[\Phi\left(G S^{\bullet}\right)\left[\phi\left(G S^{\iota}\right)\right]^{-1}\right]=\Phi\left[\phi\left(G S^{\star}\right)\left[\phi\left(G S^{\star}\right)\right]^{-1}\right]=\Phi(1)=1 .
$$

5.6. If $u \in \mathfrak{u}$, then $\Phi(u)=1$.

Proof. By the definition of $\mathfrak{u}$, we have for $u$ a formula of the type

$$
u=U_{i_{1}}^{\epsilon_{1}} \cdots U_{i_{r}}^{\epsilon_{r}}
$$

If $r=1$, then $\Phi(u)=1$ by 5.5 . Proceeding by induction, we can thus assume that 


$$
\Phi\left(U_{i_{1}}^{\epsilon_{1}} \cdots U_{i_{r-1}}^{\epsilon_{r-1}}\right)=1
$$

By $5.4,5.5$ it follows that

$$
\Phi(u)=\Phi\left[\Phi\left(U_{i_{1}}^{\epsilon_{1}} \cdots U_{i_{r-1}}^{\epsilon_{r-1}}\right) U_{i_{r}}^{\epsilon_{r}}\right]=\Phi\left(U_{i_{r}}^{\epsilon_{r}}\right)=1 .
$$

5.7. If $i \neq j$, then $g_{i}$ and $g_{j}$ are in different left cosets relative to $\mathfrak{u}$.

Proof. Suppose that $g_{i}=u g_{j}, u \in \mathfrak{U}$. By 5.3, 5.4, 5.6 we have then

$$
g_{i}=\Phi\left(g_{i}\right)=\Phi\left(u g_{j}\right)=\Phi\left[\Phi(u) g_{j}\right]=\Phi\left(g_{j}\right)=g_{j},
$$

a contradiction.

5.8. $\Phi\left(G S^{\epsilon}\right)=\phi\left(G S^{\epsilon}\right)$.

Proof. This fact has been established in the course of the proof in 5.5.

5.9. The preceding results may be summarized as follows. Each left coset relative to the subgroup $\mathfrak{U}=\mathfrak{U}[\{G\}, \phi(H)]$ contains exactly one element of the given Schreier system $g_{1}, \cdots, g_{i}, \cdots$ (see 5.7, 5.2, 5.1). Thus $\mathfrak{U}$ and the given Schreier system are associated in the sense of 1.2. If from each left coset we select as a representative the unique $G$ that lies in it, then 5.2 means that $\Phi(f)$ is simply the representative of the left coset that contains $f$ (cf. 5.1). In view of 5.6, $\mathfrak{U}$ itself as a left coset has the representative $1=g_{1}$. Thus $f \in \mathfrak{U}$ if and only if $\Phi(f)=1$. In particular, it follows that $\Phi(f)$ coincides with the $\Psi(f)$ defined in 2.4. Hence 5.8 means that our $\phi(H)$ is associated with the subgroup $\mathfrak{U}=\mathfrak{U}[\{G\}, \phi(H)]$ in the sense of 2.5 .

6. Generality of the subgroup $\mathfrak{U}[\{G\}, \phi(H)]$.

6.1. Given in $\mathfrak{F}$ a Schreier system $g_{1}, \cdots, g_{i}, \cdots$ that satisfies the condition (C) (see 2.7), we already noted (see 4.5) that the construction described in 4.1 yields the most general function $\phi(H)$ admissible for the given Schreier system. Once an admissible function $\phi(H)$ has been selected, the subgroup $\mathfrak{U}=\mathfrak{U}[\{G\}, \phi(H)]$ is univocally determined by means of the (free) generators $U_{1}, U_{2}, \cdots$ (see 3.15). We propose to verify that we obtain every subgroup of $\mathfrak{F}$ in the form $\mathfrak{U}=\mathfrak{U}[\{G\}, \phi(H)]$ if we use the freedom allowed by our construction. To establish this fact, let $\mathfrak{U}^{*}$ be any subgroup of $\mathfrak{F}$. From each left coset relative to $\mathfrak{U}^{*}$, we select a representative in such a manner that these representatives $g_{1}, \cdots, g_{i}, \cdots$ form a Schreier system (see 1.1). We shall write

$$
f_{1} \sim f_{2}\left(\bmod \mathfrak{u}^{*}\right)
$$

to state that the elements $f_{1}, f_{2}$ of $\mathfrak{F}$ are in the same left coset relative to $\mathfrak{U}^{*}$. Thus for every $f \in \mathfrak{F}$ there exists a unique subscript $i$ such that

$$
f \sim g_{i}\left(\bmod \mathfrak{u}^{*}\right) \text {. }
$$

This unique $g_{i}$ will be denoted by $\Psi^{*}(f)$. Using again $G, S, H$ as generic notations in the sense of 2.2 , we define 


$$
\phi(H)=\Psi^{*}(H) .
$$

According to $2.4, \phi(H)$ is then admissible for the Schreier system $g_{1}, \cdots$, $g_{i}, \cdots$, and hence we have a corresponding group $\mathfrak{U}[\{G\}, \phi(H)]$ (see 3.15). We propose to show that

$$
\mathfrak{U}^{*}=\mathfrak{u}[\{G\}, \phi(H)] .
$$

Proof. Let us write $\mathfrak{U}$ for $\mathfrak{U}[\{G\}, \phi(H)]$. Using the symbol $\Phi(f)$ in the sense of 5.1, we know by 5.9 that $f \in \mathfrak{U}$ if and only if $\Phi(f)=1$. On the other hand, since $g_{1}=1$, we have $f \in \mathfrak{U}^{*}$ if and only if $\Psi^{*}(f)=1$. Thus (2) is established if we can show that

$$
\Phi(f)=\Psi^{*}(f)
$$

for every $f \in \mathfrak{F}$. Now (3) is obvious if $f=1$. So we can assume that $f \neq 1$. Let then

$$
f=S_{j_{1}}^{\epsilon_{1}} \cdots S_{j_{r}}^{\epsilon_{r}}
$$

be any element of $\mathfrak{F}$, written in reduced form. We shall use induction with respect to the length $r$ of $f$.

Step 1. $r=1$. Then $f=S_{j_{1}}^{\epsilon_{1}}$, and by 5.1 we have $\Phi(f)=\phi\left(1 \cdot S_{j_{1}}^{c_{1}}\right)$. In view of (1) it follows that $\Phi(f)=\Psi^{*}(f)$.

Step 2. Assume now that (3) has been established for elements whose length does not exceed $r-1$. Applying the $W$-process (see 5.1) to the $f$ given by (4), we obtain in view of (1):

$$
\begin{gathered}
W_{r-1} \equiv \Phi\left(S_{j_{1}}^{\epsilon_{1}} \cdots S_{j_{r-1}}^{\epsilon_{r-1}}\right) \equiv \Psi^{*}\left(S_{j_{1}}^{\epsilon_{1}} \cdots S_{j_{r-1}}^{\epsilon_{r-1}}\right), \\
\Phi(f) \equiv W_{r} \equiv \phi\left(W_{r-1} S_{j_{r}}^{\epsilon_{r}}\right) \equiv \Psi^{*}\left(W_{r-1} S_{j_{r}}^{\epsilon_{r}}\right) .
\end{gathered}
$$

Now by the definition of $\Psi^{*}$ the relation (5) implies the existence of an element $u^{*} \in \mathfrak{U}^{*}$ such that

$$
W_{r-1}=u^{*} S_{j_{1}}^{\epsilon_{1}} \cdots S_{j_{r-1}}^{\epsilon_{r-1}}
$$

and hence we have

$$
W_{r-1} S_{j_{r}}^{\epsilon_{r}}=u^{*} f, \quad W_{r-1} S_{j_{r}}^{\epsilon_{r}} \sim f\left(\bmod \mathfrak{u}^{*}\right)
$$

Consequently (cf. (1))

$$
\Psi^{*}(f)=\Psi^{*}\left(W_{r-1} S_{j_{r}}^{e_{r}}\right)=\phi\left(W_{r-1} S_{j_{r}}^{e_{r}}\right)=W_{r}=\Phi(f)
$$

and (3) is proved.

6.2. According to our results, for every Schreier system $g_{1}, \cdots, g_{i}, \ldots$ that satisfies the condition (C) of 2.7 we have at least one associated sub: 
group $\mathfrak{U}$. There arises the question whether we always have an invariant subgroup associated with such a Schreier system. The answer is in the negative. As an example, let us take $\mathfrak{F}$ as the free group over the (free) generators $a, b$, and let us consider the Schreier system

$$
1, a, b, a b, b a \text {. }
$$

Let $\phi(H)$ be any function admissible for the system (1), and let $\mathfrak{U}$ be the corresponding group $\mathfrak{U}[\{G\}, \phi(H)]$. We assert that $\mathfrak{U}$ is not an invariant subgroup of $\mathfrak{F}$. Indeed, let us assume that $\mathfrak{U}$ is invariant, and let us denote by (S) the factor group $\mathfrak{F} / \mathfrak{U}$. Then we have a homomorphism $T(\mathfrak{F})=\mathbb{B}$ such that

$$
f \in \mathfrak{U} \text { if and only if } T(f)=1 \text {. }
$$

Since the Schreier system (1) contains five elements, it follows by 5.9 that the index of $\mathfrak{U}$ is equal to five, and hence $\mathbb{B}$ is the cyclic group of order five. Thus (S) is commutative. Hence on setting

$$
T(a)=\alpha, \quad T(b)=\beta,
$$

we have the relations

$$
T\left(a b a^{-1} b^{-1}\right)=T(a) T(b) T(a)^{-1} T(b)^{-1}=\alpha \beta \alpha^{-1} \beta^{-1}=1,
$$

and consequently, by (2), $a b a^{-1} b^{-1} \in \mathfrak{U}$. It follows that $a b$ and $b a$ are in the same left coset relative to $\mathfrak{U}$, in contradiction with the fact (see 5.9) that no two elements of a Schreier system $\{G\}$ are in the same left coset relative to the subgroup $\mathfrak{U}=\mathfrak{U}[\{G\}, \phi(H)]$. Let us recall that every finite Schreier system satisfies the condition (C) (see 2.7), and hence our example is valid.

\section{Shortest Schreier systems.}

7.1. From this point on we assume that the given free group $\mathfrak{F}$ has finite rank: $\rho(\mathfrak{F})=n<+\infty$. Accordingly, $\mathfrak{F}$ is given by a set of $n$ free generators $S_{1}, S_{2}$, $\cdots, S_{n}$. Part of the following discussion would apply equally well in the case $\rho(\mathfrak{F})=\infty$, but to achieve uniformity of presentation we prefer to assume throughout that the rank of $\mathfrak{F}$ is finite.

7.2. Let $\mathfrak{U}$ be a subgroup of $\mathfrak{F}$. By 6.1 we can obtain $\mathfrak{U}$ in the form $\mathfrak{U}$ $=\mathfrak{U}[\{G\}, \phi(H)]$, where $\phi(H)=\Psi(H)$ (see 1.1, 2.2). We propose to derive criteria for the Schreier system $\{G\}=\left(g_{1}, \cdots, g_{i}, \cdots\right)$ to be shortest relative to $\mathfrak{U}$ (see 1.3).

7.3. Obviously, $\{G\}$ is shortest relative to $\mathfrak{U}$ if and only if $l[\Psi(f)] \leqq l(f)$ for every $f \in \mathfrak{F}$ (see $1.3,1.1$ ). Thus the condition

$$
l[\phi(H)] \leqq l(H) \text { for every } H
$$

is surely a necessary condition for $\{G\}$ to be shortest relative to $\mathfrak{u}$, since $\dot{\phi}(\dot{H})=\Psi(H)$. We a ssert that (1) is also sufficient.

Proof. Suppose that (1) holds. Let $f=S_{i_{1}}^{\epsilon_{1}} \cdots S_{t_{r}}^{e_{r}}$ be any element of $\mathfrak{F}$ in reduced form. Applying to $f$ the $W$-process (see 5.1), we obtain (cf. 5.9) 
$W_{0} \equiv 1, W_{1} \equiv \phi\left(W_{0} S_{i_{1}}^{\epsilon 1}\right), W_{2} \equiv \phi\left(W_{1} S_{i_{2}}^{\epsilon_{2}}\right), \cdots, W_{r} \equiv \phi\left(W_{r-1} S_{i_{r}}^{\epsilon_{r}}\right) \equiv \Phi(f) \equiv \Psi(f)$.

Let us write $l_{j}=l\left(W_{j}\right), j=0,1, \cdots, r$. Then $l_{0}=l\left(W_{0}\right)=0$, and for $j>1$ we have by (1):

$$
l_{j}=l\left[\phi\left(W_{j-1} S_{i_{j}}^{\epsilon_{j}}\right)\right] \leqq l\left(W_{j-1} S_{i_{j}}^{\epsilon_{j}}\right) \leqq l\left(W_{j-1}\right)+1=l_{j-1}+1 .
$$

Hence we obtain successively $l_{1} \leqq l_{0}+1=1, l_{2} \leqq 2, l_{3} \leqq 3, \cdots, l_{r} \leqq r$. Since $l_{r}=l[\Psi(f)], r=l(f)$, the proof is complete.

7.4. Given $\mathfrak{U}=\mathfrak{U}[\{G\}, \phi(H)]$ as in 7.2 , let us consider one of the elements (see 2.3)

$$
1 \neq V=G S^{\epsilon}\left[\phi\left(G S^{\epsilon}\right)\right]^{-1} .
$$

Then by 3.8, $V$ is reduced as written, and hence

$$
l(V)=l(G)+l\left[\phi\left(G S^{\epsilon}\right)\right]+1
$$

We shall say that the significant factor $S^{\epsilon}$ of $V$ (see 3.10) is centered in $V$ if one of the following two conditions holds: (i) $l(V)=2 k+1$ and the significant factor is in the position $k+1$, or else (ii) $l(V)=2 k$ and the significant factor is in the position $k$ or $k+1$.

Using this terminology, we assert that the Schreier system $\{G\}$ is shortest for $\mathfrak{U}=\mathfrak{U}[\{G\}, \phi(H)]$ if and only if the significant factor is centered in every element $V \neq 1$.

Proof. Necessity. Suppose $\{G\}$ is shortest for $\mathfrak{u}$, and take any $V$ as in (1). Put $G_{1}=\phi\left(G S^{\epsilon}\right)$. Then (see 2.3)

$$
\phi\left(G_{1} S^{-\epsilon}\right)=\phi\left[\phi\left(G S^{\epsilon}\right) S^{-\epsilon}\right]=G .
$$

Also, $V$ is reduced as written by 3.8 . Hence if we put $\lambda=l(G), \lambda_{1}=l\left(G_{1}\right)$, we have (since $\{G\}$ is shortest by assumption)

$$
\begin{aligned}
\lambda_{1} & =l\left(G_{1}\right)=l\left[\phi\left(G S^{\epsilon}\right)\right] \leqq l\left(G S^{\epsilon}\right)=\lambda+1, \\
\lambda & =l(G)=l\left[\phi\left(G_{1} S^{-\epsilon}\right)\right] \leqq l\left(G_{1} S^{-\epsilon}\right)=\lambda_{1}+1 .
\end{aligned}
$$

Consequently

$$
\left|\lambda-\lambda_{1}\right| \leqq 1 \text {. }
$$

From (2) and (4) we infer that $l(V)=\lambda+\lambda_{1}+1$ and hence

$$
l(V)=\left\{\begin{array}{lll}
2 \lambda & \text { if } \quad \lambda_{1}=\lambda-1 \\
2 \lambda+1 & \text { if } \quad \lambda_{1}=\lambda \\
2 \lambda+2 & \text { if } \quad \lambda_{1}=\lambda+1
\end{array}\right.
$$

Furthermore, the significant factor is in the position $\lambda+1$, and hence it is centered in view of (5).

Sufficiency. Let us now suppose that the significant factor is centered in 
every $V \neq 1$. To prove that $\{G\}$ is shortest, we have to verify the condition (1) in 7.1. So let us take any product GSe. We proceed to show that

$$
l\left[\phi\left(G S^{\epsilon}\right)\right] \leqq l\left(G S^{\bullet}\right) .
$$

If $G S^{\epsilon}$ is equal to some $g_{i}$, then $\phi\left(G S^{\epsilon}\right)=g_{i}=G S^{\epsilon}$ and (6) is obvious. Otherwise, the expression

$$
V=G S^{\epsilon}\left[\phi\left(G S^{\epsilon}\right)\right]^{-1}
$$

is not equal to 1 by 3.4 and is reduced as written by 3.8 . By assumption, the significant factor $S^{\epsilon}$ is centered in $V$.

Case 1. $l(V)=2 k+1$. Then $S^{\epsilon}$ must be in the position $k+1$, and thus

$$
l(G)=l\left[\phi\left(G S^{\epsilon}\right)\right]=k .
$$

Since $V$ is reduced as written, it follows that

$$
l\left(G S^{\epsilon}\right)=l(G)+1=k+1>l\left[\phi\left(G S^{\epsilon}\right)\right],
$$

and thus (6) holds.

Case 2. $l(V)=2 k$. Then $S^{e}$ must be either in the position $k$ or else in the position $k+1$. In the first case we have $l(G)=k-1, l\left[\phi\left(G S^{\epsilon}\right)\right]=k$, and hence (since $V$ is reduced as written)

$$
l\left(G S^{\epsilon}\right)=l(G)+1=k=l\left[\phi\left(G S^{\epsilon}\right)\right] .
$$

In the second case, we have similarly $l(G)=k, l\left[\phi\left(G S^{\epsilon}\right)\right]=k-1$, and hence

$$
l\left(G S^{\mathrm{e}}\right)=l(G)+1=k+1>l\left[\phi\left(G S^{\mathrm{e}}\right)\right] .
$$

Thus (6) holds in all cases.

7.5. Given a subgroup $\mathfrak{U}$ of $\mathfrak{F}$ in the form $\mathfrak{U}=\mathfrak{U}[\{G\}, \phi(H)]$, suppose that $\{G\}$ is shortest relative to $\mathfrak{U}$. Consider an element (cf. 2.3)

$$
\begin{array}{rlrl}
f & =V_{1} V_{2} \cdots V_{r} ; & V_{i} V_{i+1} \neq 1, & i=1, \cdots, r-1 ; \\
V_{i} & \neq 1, & i=1, \cdots, r .
\end{array}
$$

We assert that (cf. 1.3)

$$
l(f) \geqq \max \left[l\left(V_{1}\right), l\left(V_{2}\right), \cdots, l\left(V_{r}\right)\right] .
$$

Proof. The assertion is obvious if $r=1$. Proceeding by induction, we assume that

$$
l\left(V_{1} \cdots V_{r-1}\right) \geqq \max \left[l\left(V_{1}\right), \cdots, l\left(V_{r-1}\right)\right] .
$$

Since each $V$ is written, by our general agreement, in reduced form, cancellations in the product $V_{1} V_{2} \cdots V_{r}$ spread from between adjacent factors and never reach the significant factors of the $V$ 's (see 3.11). Suppose that the last $t$ factors of $V_{r-1}$ cancel with the first $t$ factors of $V_{r}$. Since the significant factors are centered in each $V$ by 7.4 , we have the inequalities 


$$
l\left(V_{r-1}\right) \geqq 2 t, \quad l\left(V_{r}\right) \geqq 2 t .
$$

Obviously

$$
l\left(V_{1} \cdots V_{r}\right)=l\left(V_{1} \cdots V_{r-1}\right)+l\left(V_{r}\right)-2 t .
$$

From (3), (4), and (5) we infer that

$$
\begin{aligned}
& l\left(V_{1} \cdots V_{r}\right) \geqq \max \left[l\left(V_{1}\right), \cdots, l\left(V_{r-1}\right)\right], \\
& l\left(V_{1} \cdots V_{r}\right) \geqq l\left(V_{r-1}\right)-2 t+l\left(V_{r}\right) \geqq l\left(V_{r}\right) .
\end{aligned}
$$

Clearly, (6) and (7) yield (2).

7.6. In preparation for an application of the preceding results on shortest Schreier systems, we insert here some general comments on subgroups $\mathfrak{U}$ of $\mathfrak{F}$. By assumption, $\mathfrak{F}$ is given in terms of a finite system of free generators $S_{1}, S_{2}, \cdots, S_{n}$. Every subgroup of $\mathfrak{F}$ is again a free group (see Reidemeister [1]); incidentally, an independent proof of this fact follows from 3.15, 6.1 of the present paper. Let then $\{\alpha\}=\left(\alpha_{1}, \cdots, \alpha_{j}, \cdots\right)$ and $\{\beta\}=\left(\beta_{1}, \cdots\right.$, $\left.\beta_{j}, \cdots\right)$ be any two systems of free generators of the subgroup $\mathfrak{U}$. Let $\beta_{j}(\alpha)$ denote the reduced expression of $\beta_{j}$ in terms of the generators $\alpha$. For a given positive integer $m$, let $\alpha_{i_{1}}, \cdots, \alpha_{i_{r}}$ be those of the generators $\alpha$ that actually occur in $\beta_{1}(\alpha), \cdots, \beta_{m}(\alpha)$. Then $r \geqq m$. This fact is well known. For the convenience of the reader, we sketch the proof. Deny the assertion and assume that

$$
r<m \text {. }
$$

Let $\Omega$ be the commutator subgroup of $\mathfrak{U}$. For $\beta_{j}, j=1, \cdots, m$, we have then expressions of the form

$$
\beta_{j}=\alpha_{i_{1}}^{a_{j 1}} \alpha_{i_{2}}^{a_{j 2}} \cdots \alpha_{i_{r}}^{a_{j r}} \mathfrak{l}_{j}, \quad \mathfrak{l}_{j} \in \Omega, j=1, \cdots, m,
$$

where the exponents $a_{j t}$ are integers (positive, negative, or zero). Now let us consider the system of equations

$$
a_{1 t} x_{1}+a_{2 t} x_{2}+\cdots+a_{m t} x_{m}=0, \quad t=1, \cdots, r .
$$

From (1) we infer that we have a set of integers $x_{1}, \cdots, x_{m}$, not all zero, that solve (3). In terms of these integers, we obtain from (2) and (3) a relation of the form

$$
\beta_{1 .}^{x_{1}} \beta_{2}^{x_{2}} \cdots \beta_{m}^{x_{m}}=\mathfrak{l} \in \Omega .
$$

Since $\beta_{1}, \beta_{2}, \cdots$ are free generators of $\mathfrak{U}$, (4) implies that $x_{1}=x_{2}=\cdots=x_{m}$ $:=0$, a contradiction.

7.7. Given $\mathfrak{U}$ and $\{\alpha\}$ as in 7.6, each $\alpha$ has a length $l(\alpha)$ in terms of the generators $S_{1}, S_{2}, \cdots, S_{n}$ (see 1.3). Since the number of the generators $S$ of $\mathfrak{F}$ is finite by assumption, we have only a finite number of elements $f \in \mathfrak{F}$ whose 
lengths do not exceed an assigned positive integer. Accordingly, we can arrange the generators $\alpha$ in a (finite or infinite) sequence $\alpha_{1}, \cdots, \alpha_{j}, \cdots$ in such a manner that

$$
l\left(\alpha_{1}\right) \leqq \cdots \leqq l\left(\alpha_{j}\right) \leqq \cdots .
$$

We shall then say that the generators $\alpha$ are ordered by length. Let now $\{\beta\}$ $=\left(\beta_{1}, \cdots, \beta_{j}, \cdots\right)$ be any other system of free generators, ordered by length, of $\mathfrak{u}$. If for every such choice of $\{\beta\}$ we have

$$
l\left(\beta_{j}\right) \geqq l\left(\alpha_{j}\right), \quad j=1,2, \cdots,
$$

then we shall say that $\{\alpha\}$ is a minimal system of free generators for $\mathfrak{U}$. It is of course not obvious that such a minimal system $\{\alpha\}$ exists. For the case when the rank of $\mathfrak{u}$ is finite, the work of Nielsen [2] yields an existence proof. We shall give presently a theorem which covers the case of subgroups of infinite rank and also reveals the relationship between shortest Schreier systems and minimal systems of generators.

7.8. Let $\mathfrak{U}$ be any subgroup of $\mathfrak{F}$ (see 7.1). According to Schreier, there exists then a shortest Schreier system $\{G\}$ associated with $\mathfrak{U}$ in the sense of 1.2 (see Reidemeister [1]). In terms of this system $\{G\}$, we have for $\mathfrak{U}$ a representation $\mathfrak{U}=\mathfrak{u}[\{G\}, \phi(H)]$ by 6.1 . Let $\{U\}=\left(U_{1}, \cdots, U_{j}, \cdots\right)$ be the corresponding system of free generators (see 3.15). By 7.7 we can assume that the system $\{U\}$ is ordered by length:

$$
l\left(U_{1}\right) \leqq \cdots \leqq l\left(U_{j}\right) \leqq \cdots .
$$

We assert that $\{U\}$ is a minimal system of free generators, in the sense of 7.7.

Proof. Let $\{\beta\}=\left(\beta_{1}, \cdots, \beta_{j}, \cdots\right)$ be any set of free generators for $\mathfrak{u}$; ordered by length:

$$
l\left(\beta_{1}\right) \leqq \cdots \leqq l\left(\beta_{j}\right) \leqq \cdots \cdot
$$

We have to show that

$$
l\left(\beta_{j}\right) \geqq l\left(U_{j}\right), \quad j=1,2, \cdots .
$$

Let $\beta_{j}(U)$ denote the reduced expression of $\beta_{j}$ in terms of the generators $U_{1}, U_{2}, \cdots$. Then $\beta_{1}(U)$ contains at least one $U$, say $U_{i 1}$, with an exponent \pm 1 . Hence by 7.5 we have, in view of (1),

$$
l\left(\beta_{1}\right) \geqq l\left(U_{i_{1}}\right) \geqq l\left(U_{1}\right) .
$$

Thus (3) holds for $j=1$. If the general validity of (3) is denied, then we should have some positive integer $m>1$ such that

$$
\begin{aligned}
& l\left(\beta_{j}\right) \geqq l\left(U_{j}\right), \\
& l\left(\beta_{m}\right)<l\left(U_{m}\right) .
\end{aligned}
$$

Let now $U_{i_{1}} \cdots, U_{i_{r}}$ denote those of the generators $U$ that occur in 
$\beta_{1}(U), \cdots, \beta_{m}(U)$. We assert that

$$
i_{j_{j}} \leqq m-1,
$$

$$
j=1, \cdots, r \text {. }
$$

Indeed, $U_{i,}$ occurs by assumption, with an exponent \pm 1 , in at least one of $\beta_{1}(U), \cdots, \beta_{m}(U)$, say in $\beta_{t}(U)$. By $7.5,(5)$, (2) we have then

$$
l\left(U_{m}\right)>l\left(\beta_{m}\right) \geqq l\left(\beta_{t}\right) \geqq l\left(U_{i_{j}}\right) .
$$

By (1) it follows that $i_{j}<m$ and (6) is established. Since $i_{1}, \cdots, i_{r}$ are distinct positive integers, (6) implies that $r<m$, in contradiction with the result stated in 7.6. Thus (5) is shown to lead to a contradiction, and the proof is complete.

7.9. If we assume that $\mathfrak{U}$ is of finite rank, then the preceding result may be derived from those of Nielsen [2] as follows. Let $\mathfrak{U}=\mathfrak{U}[\{G\}, \phi(H)]$, where $\{G\}$ is shortest relative to $\mathfrak{u}$, and let $U_{1}, \cdots, U_{N}, N=\rho(\mathfrak{u})<\infty$, be the corresponding system of free generators for $\mathfrak{U}$. A simple reasoning shows then that the property established in 7.4 implies that $U_{1}, \cdots, U_{N}$ is a reduced system of generators for $\mathfrak{U}$ in a certain sense defined by Nielsen [2]. On the other hand, according to Nielsen every reduced system of free generators for $\mathfrak{U}$ is minimal in the sense of 7.7 , and the result of 7.8 follows for the case when $\rho(\mathfrak{U})<\infty$. As far as we are aware, the connection between shortest Schreier systems and minimal systems of free generators, revealed by the theorem 7.8 , is new even in the case $\rho(\mathfrak{U})<\infty$.

\section{BIBLIOGRAPHY}

1. K. Reidemeister, Einführung in die kombinatorische Topologie, Braunschweig, Vieweg, 1932.

2. J. Nielsen, Om Regning med ikke-kommutative Faktorer og dens Anvendelse $i$ Gruppeteorien, Matematisk Tidsskrift B (1921) pp. 77-94.

The Ohio State University, Columbus, Оhio. 